Review Article

\title{
A Status Report on Autonomic Nervous System: The Indian Research Scenario During the Last Five Years
}

\author{
RAMESH K GOYAL* and AJIT K THAKUR \\ Delhi Pharmaceutical Sciences and Research University, Pushp Vihar Sector-III, M.B. Road, New Delhi \\ 110017, India
}

(Received on 04 August 2017; Accepted on 20 November 2017)

\begin{abstract}
Autonomic nervous system, as complex, multi-faceted neural networks has now emerged as an important system involved in various neuropsychiatric and neurodegenerative including memory disorders in addition to cardiovascular diseases. Earlier research in auto-pharmacology was focussed on receptor characterisation and understanding mechanism involved in specific conditions. Currently, focus is more on comorbid conditions associated with complications of cardiovascular system and neurological disorders. Interestingly, there have been increased research works on metabolic disorders. Yet another facet of research is the scientific evidence of yoga for the management of comorbid disorders associated neurological disorders.
\end{abstract}

Keywords: Autonomic Nervous System; Cardiovascular Disorders; Neuropsychiatric Disorders; Metabolic Disorders; Yoga

\section{Introduction}

The autonomic nervous system is one of the key regulators for involuntary activities principally controlling below the level of consciousness to manage and synchronize bodily homeostatic functions and visceral activities under physical and mental stress. Earlier studies in the field of autonomic pharmacology were focussed on receptor characterizations and neurotransmitter control through various autacoids (Adithan, 1996; Goyal and Umrani, 2000). Autonomic nervous system outputs are based on secretory activity of glands and contraction of smooth muscles, while inputs mainly derive from afferent sensations arising from visceral receptors (Bassi and Bozzali, 2015; Nivethitha et al., 2016). Autonomic nervous system with its enteric, parasympathetic and sympathetic nervous divisions plays a key role in regulation of function associated with the central nervous system like memory, cognition, movements of various muscle in particular vasculature and secretion from glands. The role of autonomic nervous system on over visceral functions such as heart rate, respiratory rate, digestion and maintenance of internal homeostasis was known from earlier days. Apart from neurological and neuropsychiatric disorders, role of abnormal autonomic functions is implicated in comorbid conditions associated with various cardiovascular, digestive and metabolic disorders (Grassi and Ram, 2016; Pal et al., 2014a). Various symptoms like problems with the regulation of heart rate, blood pressure, body temperature, perspiration, and bowel and bladder functions are assigned to alterations in autonomic controls. In last couple of years, more attention has been paid at the cardiovascular system disorders, brain disorders and metabolic disorders associated with autonomic nervous system (Indumathy et al., 2015; Kumar et al., 2017; Nayani et al., 2016; Pal et al., 2014a; Senan and Petrosyan, 2014; Udupa et al., 2007). It is now evident that autonomic nervous system plays a vital role in attention, self-regulation, emotional stability and social affiliation (Rukmani et $a l ., 2016)$. Its failure is commonly observed in many neurological and neuropsychiatric disorders, including neurodegenerative and vascular brain diseases, memory impairment, spinal cord injury, and peripheral

*Author for Correspondence: E-mail: goyalrk@gmail.com 
neuropathies, where they trigger or enhance pathological symptoms (Bansal et al., 2011; Jangir et al., 2016; Shaikh and Verma, 2011; Tota et al., 2012b).

Literature searches were done from PubMed, ScienceDirect and Google Scholar. These literature searches were filtered for the last five years and further publications were selected only for the research works which were published from Indian laboratories/Research centres by the Indian authors/ co-authors. In addition, we have reviewed the research works reported on yoga and its beneficial effect/s on abnormal autonomic functions.

\section{Autonomic Nervous System and Cardiovascular System Disorders}

Cardiovascular functions are controlled by neural factors, temperature and various hormones, and of these, neural factors primarily concern the autonomic nervous system, which play a major role in maintaining and regulating cardiac functions, e.g., blood pressure and heart rate (Nivethitha et al., 2016). Autonomic cardiovascular control is impaired in hypertension, leading to a reduction in the parasympathetic tone and to an increase in the sympathetic influences to the heart and peripheral vessels. The sympathetic dysfunction depends on a variety of reflex and nonreflex mechanisms and participates at development and progression of the essential hypertensive state (Grassi and Ram, 2016). An experimental study was conducted to evaluate the role of sympathetic nervous system and oxidative stress in hemodynamic and autonomic control after acute inhibition of the synthesis of nitric oxide, using intravenous (i.v.) injection of 30 $\mathrm{mg} / \mathrm{kg} \mathrm{N}(\mathrm{G})$-nitro-l-arginine methyl ester in adult Wistar rats. It has been concluded that even though pressor response was unaffected, reversal of cardiac autonomic responses and decline in oxidative stress following sympathectomy in N(G)-nitro-l-arginine methyl ester -treated rats reflects a significant role for sympathetic innervation in acute $\mathrm{N}(\mathrm{G})$-nitro-1arginine methyl ester -induced hypertension (Chaswal et al., 2011).

The relationship has been found between sudden cardiac death and autonomic disbalance of nervous system, which affect on baro-reflector regulation of the heart rhythm. The importance of heart rate in cardiovascular prognosis can be explained by its relationship with major pathophysiological determinants. In a prospective study, researchers have revealed a higher risk for sudden death for those apparently healthy individuals whose heart rate responses are exaggerated under mild emotional stress and below normal during peak exercise. Therefore, the individuals showing these symptoms required preventive strategies to reduce the probability of ischemic heart disease (Senan and Petrosyan, 2014).

Under normal conditions, parasympathetic and sympathetic nervous systems interact to regulate the heart rate of about 70 beats per minute. Activation of sympathetic nervous system by emotional or physical stress increases heart rate and the force of heart beat. A cross-sectional study was carried on 134 rice mill workers and found that the rice mill workers are under high level of dust exposure which has abnormalities seen in cardiovascular system and deleterious effects on their blood and tissues due to high oxidative stress (Patil et al., 2015).

Heart rate, autonomic nervous system disorders and diseases like diabetes affect the corrected QT (QTc) interval. However, the effect of exercise that alters autonomic nervous system activity, on QTc is not clear. On the other hand, incidence of sudden cardiac death increases many-fold post exercise. Recently an interesting observation was documented from a study with twenty-nine healthy medical students. From this study, authors have justified that exercise brings about detectable change in the QTc interval after a period of $5 \mathrm{~min}$ isometric exercise in normal individuals; which, in high risk individuals may predispose to sudden cardiac death (Meher et al., 2014).

Chronic mitral regurgitation is characterized by adverse ventricular re-modelling and progressive left ventricular dysfunction leading to heart failure. Some $\beta$-blockers improve left ventricular remodelling and prognosis in patients with heart failure. In a study with 100 patients of chronic rheumatic mitral regurgitation were subjected to metoprolol or placebo. The results showed beneficial effect of Metoprolol in rheumatic mitral regurgitation, targeting the sympathoadrenergic axis and exerted favourable effects on left ventricular volumes, left ventricular end-systolic stress, and left ventricular work. Further authors have warranted that more studies are required to elucidate the role of $\beta$-blockers in rheumatic mitral regurgitation (Sahoo et al., 2016). 
Acute and chronic stress is a risk factor for the development and progression of coronary artery disease. Increased arterial stiffness is an independent marker for cardiovascular disease. Cold pressor test is known to be associated with substantial activation of the autonomic nervous system. In a double-blind, placebo-controlled, crossover study, Phyllanthus emblica extract showed a significant decrease in cold pressor stress test induced changes on aortic wave reflections in healthy human subjects (Fatima et al., 2014).

Hypertension, one of the modifiable risk factors for cardiovascular disease, is known to be associated with increased oxidative stress and reduced cardiovagal modulation. Similar to hypertension, prehypertension is associated with an increased risk of adverse cardiovascular events. A hypertension screening camp study concluded that pre-hypertension in young adults is associated with increased oxidative stress and altered cardiovagal modulation. The risk factors for cardiovascular diseases in pre-hypertensive young adults were found to be equivalent to that of middle-aged adults who are in the twilight zone for developing cardiovascular dysfunctions (Thiyagarajan et al., 2013).

Fibromyalgia is generally associated with dysautonomia and amitriptyline is the most common drug prescribed for the treatment of fibromyalgia. In a preliminary study, low dose $(10 \mathrm{mg})$ of amitriptyline was studied with autonomic function tests and blood flow measurements in well-diagnosed patients with fibromyalgia. Authors have warranted that amitriptyline therapy (10mg for 3 months) increases blood flow to the affected sites. It does not affect autonomic tone and reactivity in the patients with fibromyalgia (Kulshreshtha et al., 2012a; b). Current understanding of autonomic nervous system abnormalities in fibromyalgia has been reviewed elsewhere. They have concluded that a high peripheral sympathetic tone causes regional ischaemia, which in turn results in a widespread pain. Thus, therapeutic interventions (e.g. exercise) that result in vasodilatation and favourable autonomic alterations have proven to be effective (Kulshreshtha and Deepak, 2013).

In a separate prospective randomized controlled trial study, authors suggested that passive listening to Indian music along with conventional lifestyle modifications has a role in normalizing blood pressure through autonomic function modification and thus can be used as a complementary therapy along with the other lifestyle modifications (Kunikullaya et al., 2015).

\section{Autonomic Nervous System and Brain Disorders}

There is an autonomic dysfunction in children with attention deficit hyperactivity disorder, which is a common childhood neuropsychiatric disorder. In a study, Rukmani et al. (2016) have unravelled the nature of cardiac autonomic dysfunction present in children with attention deficit hyperactivity disorder and showed that autonomic dysfunction causes heart rate variability, which is a non-invasive marker of autonomic nervous system in attention deficit hyperactivity disorder with sympathovagal imbalance and sympathetic dominance. In addition, various psychiatric disorders such as anxiety disorders, depression and schizophrenia are associated with reduction in overall heart rate variability with sympathovagal imbalance with sympathetic dominance (Udupa et al., 2007). It has been reported that abnormal heart rate variability is found in schizophrenia patients, suggesting a pathophysiological link between central autonomic dysfunction and symptoms of schizophrenia and that, these could be heritable. A study was conducted to evaluate cardiac autonomic response to mental arithmetic stress in first-degree relatives of schizophrenia patients. The authors have suggested poor recovery from cardiac autonomic functions alterations induced by arithmetic stress may be a heritable trait marker of schizophrenia (Abhishekh et al., 2014). In an independent study, authors have investigated the relationship between heart rate variability measures and in the patients suffering from depression (Jangpangi et al., 2016). They found that there was alteration in heart rate variability present in patients with depression due to enhanced sympathetic activation relative to parasympathetic component, in accordance with what most of the prior studies have suggested.

Stress-induced increase in plasma corticosterone and norepinephrine levels used as markers for the function of hypothalamo-pituitary-adrenal (HPA) axis and sympathetic nervous system respectively. Some herbal drugs and secondary metabolites have been reported to have anti-stress activity and normalise stress-induced perturbations of serotonin, dopamine 
and its metabolites, indicating modulation of brain monoaminergic system probably mediated through the HPA axis (Bali and Jaggi, 2016; Krishnamurthy et al., 2013; Krishnamurthy et al., 2011; Thakur et al., 2015; Thakur et al., 2014).

Evidence indicates that inhibition of central renin angiotensin system ameliorates memory impairment in animals and humans. In an earlier experimental study, the involvement of central angiotensin converting enzyme in streptozotocin induced neurodegeneration and memory impairment was established elsewhere (Tota et al., 2012a). In a recent study, the role of central angiotensin converting enzyme in cholinergic neurotransmission, brain energy metabolism and cerebral blood flow in mice model of memory impairment induced by injection of scopolamine (Tota et al., 2012b). Authors have concluded possible interaction between angiotensin converting enzyme and cholinergic neurotransmission and that beneficial effect of perindopril can be attributed to improvement in central cholinergic neurotransmission and cerebral blood flow.

The deregulation of cholinergic system and associated neuronal damage is thought to be a major contributor to the pathophysiologic sequelae of hypobaric hypoxia-induced memory impairment. Uniquely, the muscarinic receptors also play a role in zinc uptake. In an experimental study, the mechanistic relationship between memory dysfunction and zinc homeostasis in brain was evaluated. The authors have suggested that the free chelatable zinc released during hypobaric hypoxia might play a critical role in the neuronal damage and alteration in cholinergic function associated with hypobaric hypoxia-induced memory impairment. Further, they have speculated that zinc chelation might be a potential therapy for hypobaric hypoxia-induced cognitive impairments (Udayabanu et al., 2012).

Paroxysmal sympathetic hyperactivity is a condition in which there is extreme autonomic dysregulation leading to multiple episodes of sympathetic hyperactivity. Their incidence after the traumatic brain injury in pediatric populations is a neglected circumstance. From a case study, it has been reported that the presence of paroxysmal sympathetic hyperactivity is known to produce poorer outcome in terms of overall mortality, time needed for recovery, chances of developing infections, etc. Authors have suggested that strict management guidelines, pharmacotherapy and exact incidence in pediatric population are still required (Deepika et al., 2015).

An experimental study was conducted to study the clinical manifestations, neurobehavioral, hematobiochemical, oxidative stress, genotoxicity, and histopathological changes during acrylamide toxicity in rats. Histopathological changes observed in brain include neuronal degeneration, edema, and congestion, while spinal cord revealed demyelination in low-dose group and bilateral necrosis with malacia, liquefaction of white matter, and loss of myelin from gray matter in high-dose groups. The authors have concluded that pathological alterations in brain and spinal cord are responsible for neurobehavioral changes in rats during acrylamide toxicity (Jangir et al., 2016).

Neurological manifestations of elapid snakebites include neuromuscular paralysis and cerebrovascular complications. Autonomic manifestations are observed in almost two third of patients following moderate to severe envenomation. A study on a 10-year old boy was conducted after bite of a common Indian krait and authors have concluded that posterior reversible leukoencephalopathy can be a late complication of Indian krait bite secondary to autonomic instability with systemic hypertension (Kaushik et al., 2014).

Circadian rhythm sleep disorder-advanced sleep-phase type is a relatively uncommon disorder, mostly seen among the elderly population. Impaired circadian rhythms have been reported in neurodegenerative conditions. In a study, patients of Parkinsonian syndromes, diagnosis of multiple system atrophy were made, supported by MRI findings and evidence of autonomic dysfunction. On a detailed examination, the patient was observed to have symmetrical bradykinesia and cogwheel rigidity of limbs followed by progressive supranuclear palsy (Shukla et al., 2015). Moreover, authors have warranted that more prospective assessment for circadian sleep disorders may introduce useful insights into similar associations.

\section{Autonomic Nervous System and Metabolic Disorders}

Autonomic dysfunction can occur as a secondary 
condition of another disease process, like obesity \& diabetes. Obesity is associated with varied combinations of abnormalities in the autonomic nervous system. A strong association between falls and syncope in elderly subjects with post-prandial hypotension has been established. However, postprandial hypotension has received less attention, although it occurs more frequently than orthostatic hypotension and represents substantial cause for morbidity and mortality (Ambarish et al., 2005). In a recent comparative study of pre and post-prandial blood pressure recordings in obese and non-obese young adults, it has been concluded that post-prandial hypotension was significantly higher in obese than in non-obese patients (Sequeira and Rosario, 2016).

Altered endocrinal and autonomic nervous system responses to stress may link impaired intrauterine growth with later cardiovascular disease in gestational diabetic mothers. It has been reported that maternal diabetes during pregnancy is associated with higher cardiosympathetic stress responses in the offspring, which may contribute to their higher cardiovascular disease risk (Krishnaveni et al., 2015). Demyelination of the autonomic nervous system occurs early in vitamin $B_{12}$ deficiency and the impact of maternal vitamin $B_{12}$ deficiency during pregnancy on neuronal function in the offspring is poorly documented. However, in a birth cohort study, it has been reported that children born to mothers with a lower vitamin $B_{12}$ status have a reduced cardiac sympathetic activity. However, long-term implication of this needs to be evaluated by follow-up studies (Sucharita et al., 2014).

Pathophysiological mechanisms and role of sympathovagal imbalance in the prediction of abnormalities in cardiovascular functions in obesity has been revealed for the first time by Indumathy et al. (2015). They have concluded that sympathovagal imbalance in obesity is due to both increased sympathetic and decreased vagal activity. Abnormal cardiovascular parameters in obesity are linked to sympathovagal imbalance, which is contributed by insulin resistance, dyslipidaemia and low-grade inflammation. Low frequency to high frequency ratio of heart rate variability predicts abnormal cardiovascular parameters in obesity. In the same year, another study suggested that blood pressure response to cold pressor test was reduced in obese compared to non-obese subjects indicating reduced sympathetic activity in healthy obese adults (Grewal et al., 2015). It was found that postmenopausal women who had significantly decreased values of heart rate variability tests also had altered lipid profile. These findings showed that the changes in sex hormone levels, after menopause may alter the autonomic nervous system response and lipid metabolism and further suggesting that dyslipidaemia may be an important factor for development of autonomic dysfunction with decreased sympathovagal activity (Chaudhuri et al., 2012a; Chaudhuri et al., 2012b). In a recent study, the link of hyperlipidemia, inflammation and oxidative stress to cardiovascular risks in subclinical hypothyroidism was explored. The authors have concluded that sympathovagal imbalance is associated with subclinical hypothyroidism. Though dyslipidemia, inflammation and oxidative stress contributed to decrease baro-reflex sensitivity, subclinical hypothyroidism per se contributed maximally to it. Decreased baro-reflex sensitivity could be a physiological basis of increased cardiovascular risks in patients with sub-clinical hypothyroidism (Syamsunder et al., 2017).

A partial loss of beta-cell mass and beta-cell dysfunction in type 2 diabetes mellitus is associated with amyloid deposition but whether it is causal or consequential is debated. In type 2 diabetes, the sympathetic inputs are known to increase which could result in suppression of the secretion process. The increase in the retention time due to this suppression can allow polymerization. Moreover, in a prediabetic state, parasympathetic stimulation increases $\beta$-cell proliferation. Therefore, a combination of contrasting actions of sympathetic and parasympathetic systems could lead to increase in the amyloid deposition (Watve et al., 2014). Insulin secretion is generally regulated by sympathetic and parasympathetic nervous systems and modulates the concentrations of hypothalamic neuropeptides and monoaminergic neurotransmitters, and, in turn, hypothalamic monoamines regulate the secretion of insulin by pancreatic beta cells. A lesion of the ventromedial hypothalamus produces all the features of metabolic syndrome including insulin resistance and hyperinsulinemia (Das et al., 2011).

Although cardiovascular risks are reported in first-degree relatives of type 2 diabetics, effects of gender on sympathovagal imbalance and 
cardiovascular risks in these subjects have not been investigated. However, a multiple regression analysis study demonstrated that sympathovagal imbalance is more intense in male first-degree relatives of type 2 diabetics, and sympathovagal imbalance is associated with increased cardiovascular risk due to insulin resistance, dyslipidemia, inflammation, and oxidative stress in these subjects (Pal et al., 2014a).

Autonomic neuropathy is a risk predictor for sudden cardiac death in rheumatoid arthritis and ankylosing spondylitis. The autonomic neuropathy can be assessed by cardiovascular autonomic reflex tests according to Ewing, heart rate response to deep breathing, standing, and Valsalva tests, blood pressure response to standing and handgrip tests and Sudomotor function can be assessed by Sudoscan. In an openlabel, 12-week pilot study in 60 patients, synthetic disease-modifying anti-rheumatic drug showed improved parasympathetic and sudomotor dysfunction in both disease-modifying anti-rheumatic drug-naive rheumatoid arthritis and ankylosing spondylitis patients (Syngle et al., 2015).

Hyperthyroidism is known to increase food intake and central administration of thyroid hormone shows acute orexigenic effects and T3 influences appetite and glucose homeostasis by modulating circulating ghrelin, an important orexigenic hormone. In an experimental study, Zucker fatty rats demonstrated acute orexigenic and hyperglycemic effects of T3 which is associated with ghrelin secretion and activity. This effect seems to be mediated via vagus nerves, and is independent of glucoregulatory hormones (Patel et al., 2014). In a study with psoriatic arthritis patients, Syngle et al. (2013) have concluded that cardiovascular autonomic and peripheral sympathetic neuropathy occurs in psoriatic arthritis. Parasympathetic function is more commonly found to be abnormal than sympathetic function. However, parasympathetic function is more commonly found to be abnormal than sympathetic function in psoriatic arthritis patients.

Altered cardiac autonomic function measured by heart rate variability is observed in early stages of diabetes but the relationship between impaired fasting glucose and heart rate variability was not clear. However, from a cross-sectional blinded study, it was proved that continuous interplay between the altered cardiac autonomic function, hyperinsulinemia, insulin resistance, oxidative stress parameters, inflammatory response, and impaired fasting glucose in which one factor perpetuates another leading to the progression of disease (Thiyagarajan et al., 2012). In an independent study, cardiovascular autonomic dysfunction was found in type 2 diabetes mellitus and essential hypertension in a South Indian population (Hazari et al., 2012). Though cardiovascular risks are reported in first-degree relatives of type 2 diabetics, the pathophysiological mechanisms contributing to these risks are not known. In a multiple-regression analysis during an investigation in type 2 diabetic subject, the association of sympathovagal imbalance with risks in these subjects was established. Further, authors have concluded that sympathovagal imbalance in first-degree relatives of type 2 diabetics occurs due to sympathetic activation and vagal withdrawal. The sympathovagal imbalance contributes to pre-hypertension status and cardiovascular risks caused by insulin resistance, dyslipidemia, inflammation and oxidative stress in first-degree relatives of type 2 diabetics (Pal et al., 2013b).

\section{Autonomic Nervous System and Miscellaneous Disorders}

During an observational study in a tertiary care centre of urban India, patients with advanced gastrointestinal and pelvic malignancies were treated with neurolytic blocks of the sympathetic axis (ultrasonography-guided neurolysis of celiac plexus, superior hypogastric plexus and ganglion impar) to overcome intolerable side effects of oral morphine and patient dissatisfaction. Further, authors have suggested that ultrasonographyguided sympathetic axis neurolysis may be employed early in patients with incurable abdominal or pelvic cancer (Bhatnagar et al., 2012).

Autonomic dysfunction is common in children with Rett syndrome. A study was conducted in a tertiary care teaching hospital in north India for more than a six-month period to evaluate cardiovascular autonomic dysfunction. It was found that children with Rett syndrome exhibited significant cardiovascular autonomic dysfunction in the form of sympathetic overactivity, parasympathetic underactivity, and sympathovagal imbalance (Kumar et al., 2017).

Sudomotor dysfunction is well established in type 
2 diabetes mellitus and reflects small fibre neuropathy, cardiovascular autonomic neuropathy and peripheral sympathetic autonomic neuropathy. In a crosssectional study, association of sudomotor function with ankle-brachial index and C-reactive protein in type 2 diabetes mellitus patients was explored. Authors have concluded that sudomotor dysfunction is associated with significant peripheral artery disease, vascular inflammation and impaired glycaemic status (Chahal et al., 2017). Cushing's syndrome is associated with increased cardiovascular morbidity and mortality. It is also associated with other cardiac risk factors like hypertension, diabetes mellitus and obesity. Cardiovascular autonomic function impairment could predict cardiovascular morbidity and mortality. In a study with 25 patients with Cushing's syndrome underwent a battery of cardiovascular autonomic function tests including deep breath test, Valsalva test, handgrip test, cold pressor test and response to standing from lying position. This study showed that chronic endogenous hypercortisolism in Cushing's is associated with an impaired sympathetic cardiovascular autonomic functioning (Jyotsna et al., 2011).

A study was conducted to assess the sympathovagal balance in patients with polycystic ovary syndrome and found that polycystic ovary syndrome patients have altered autonomic modulation in the form of increased sympathetic and decreased parasympathetic reactivity and heart rate variability and this sympathovagal imbalance exposes them to cardiovascular morbidities (Saranya et al., 2014). Some of the physiological factors and athletic performance might show variation along the phases of menstrual cycle. The alterations seen in these physiological parameters of various systems relating to oscillations in hormonal levels do affect the autonomic nervous system and metabolic functions. In a prospective study (consecutively for two menstrual cycles in all three phases), authors have suggested that cyclical variation in endogenous reproductive hormones increases the muscle strength in follicular phase of the menstrual cycle (Pallavi et al., 2017).

Duchenne muscular dystrophy is a hereditary neuromuscular disorder frequently associated with progressive cardiac dysfunction, and is one of the common causes of death in these children. In a cross sectional study, children suffering from Duchenne muscular dystrophy have demonstrated alteration in autonomic tone. In addition, loss of vagal tone and an increase in sympathetic tone were observed in these children (Dhargave et al., 2014).

\section{Autonomic Nervous System and Yoga}

Yoga is a science that facilitates homeostasis, an ancient way of life intended to improve the quality of life of an individual. Practice of yoga is proposed to alter the autonomic nervous system and affect the cardiovascular functioning. Exercise is considered an acceptable method for improving and maintaining physical and emotional health. Although yoga is historically a spiritual discipline, a growing body of evidence supports the belief that yoga benefits physical and mental health (Peter et al., 2015). Beneficial effects of yoga have been postulated to be due to modulation of the autonomic nervous system. It has been advised that life-style modifications such as yoga and exercise would enable achieve the sympathovagal balance and blood pressure homeostasis in prehypertensives (Muralikrishnan et al., 2012; Pal et al., 2012). The practice of yoga is gaining immense popularity with a wide range of practices. Recent research and descriptions from the ancient texts are often concurrent with regard to the effects of the practice, taking into account expected differences between modern scientific terms and those used in the original texts. The voluntarily regulated yoga breathing has distinct effects on metabolism, the autonomic nervous system, higher brain functions, and mental state. The effects of meditation on nervous system and mental state are even clearer (Telles and Singh, 2013). Meditation is a complex process involving change in cognition, memory, and social and emotional control, and causes improvement in various cardiovascular, neurological, autoimmune, and renal pathologies. Meditation also became widely used in medical and psychological treatment therapies for stress-related physical and mental disorders (Jindal et al., 2013). From an independent study, authors have reported their observations on 32 healthy adult male student volunteers who had never practiced meditation before the study. They warranted that practice of meditation produced a relaxation response even in the young adult subjects who had never practiced meditation before. The practice of meditation reduced the physiologic stress responses 
without taking away the beneficial effect of stress, namely, improved memory scores (Mohan et al., 2011).

A study was intended to assess the influence of short-term practice of yoga for a month on heart rate variability. The authors have concluded that autonomic balance tilts toward parasympathetic predominance after 1 month practice of yoga (Vinay et al., 2016) and this study was consonant with the earlier independent study put forward substantially greater parasympathetic activity in yoga practitioners (Peter et al., 2015). During earlier prospective randomized comparative trial (to assess and compare the effects of yogic training and swimming on resting heart rate variability in normal healthy young volunteers) concluded that practicing yoga seems to be the mode of exercise with better improvement in autonomic functions as suggested by resting heart rate variability (Sawane and Gupta, 2015). In a study, patients diagnosed with coronary artery disease were allowed to yogic intervention consisted of 35-40 $\mathrm{min} /$ day, 5 days a week over a period of 18 months. This study concluded that yoga therapy is beneficial for the reduction in body mass index, waist circumference, systolic and diastolic blood pressure, and heart rate i.e., a significant positive effect was observed when yoga therapy was used as an adjunct in patients with coronary artery disease ( $\mathrm{Pal}$ et al., 2013a).

A general review on the effect of various pranayama (breathing techniques) on cardiovascular and autonomic variable suggested that slow type of yogic breathing technique was reported to produce beneficial effect on cardiovascular and autonomic variables while fast breathing techniques do not produce such effects. However, different types of Pranayama techniques produce different effects and the mechanisms behind these effects are not fully understood (Nivethitha et al., 2016). Aging is generally associated with a decline in cardiovascular, autonomic function, and brain-derived neurotropic factor. Reports are scanty regarding whether yoga can improve agerelated degenerative changes in healthy active men. A study was conducted in healthy people to evaluate the role of yoga in improving age-related degenerative changes and significant decrement of cortisol, adrenocorticotropic hormone, and increment in serotonin, dopamine, and brain-derived neurotropic factor was noted following yogic practice. Moreover, authors have concluded that yogic practices are helpful in the prevention of age-related degeneration by changing cardiometabolic risk factors, autonomic function, and brain-derived neurotropic factor in a healthy male (Pal et al., 2014b).

From an investigational study on 30 normal young healthy engineering students published elsewhere reported that yoga-practising group showed significant improvement in various cognitive functions, such as performance enhancement, neural activity, attention, and executive function. It also resulted in increase in the heart rate variability, parasympathetic nervous system activity, and the balanced autonomic nervous system reactivity (Nagendra et al., 2015). In the same year, a study with 10 type 2 diabetic subjects, authors have explored the role of regular practice of yoga on cognition in type 2 diabetes and studied the relation between cognition and the functional status of autonomic nervous system by considering cardiac autonomic function tests. They have concluded that regular practice of yoga in combination with oral hypoglycemic agents has a positive effect on cognition in type 2 diabetes (Rajani et al., 2015).

Comparative cardiovascular effects of different yoga Asanas (Dhanurasana, Vakrasana, Janusirasasana, Matsyasana and Shavasana) in healthy young volunteers has been reported elsewhere (Bhavanani et al., 2014). This study also provided initial evidence of differential cardiovascular effects of Asanas and subtle differences between right and left sided performance. Further, authors have suggested that cardiovascular recovery is greater after the performance of the Asanas as compared to shavasan; thus, implying a better response when effort precedes relaxation. Apart from the various Asanas, breathing techniques are regularly recommended for relaxation, stress management, control of psychophysiological states, and to improve organ function. Yogic breathing, defined as a manipulation of breath movement, has been shown to positively affect immune function, autonomic nervous system imbalances, and psychological or stress-related disorders. Therefore, Sudarshan Kriya yoga can be a beneficial, low-risk, low-cost adjunct to the treatment of stress, anxiety, post-traumatic stress disorder, depression, stress-related medical illnesses, substance abuse, and rehabilitation of criminal offenders (Zope and Zope, 2013). A study with 30 healthy male 
volunteers showed maximum changes in autonomic variables and the breath rate during the state of effortless meditation (dhyana). The changes were all suggestive of reduced sympathetic activity and/or increased vagal modulation. During dharana, there was an increase in skin resistance. The changes in heart rate variability during ekagrata and cancalata were inconclusive (Telles et al., 2013). Effect of shortterm and long-term Brahmakumaris Raja yoga meditation on physiological variables like heart rate, respiratory rate, systolic blood pressure and diastolic blood pressure was evaluated in 100 subjects practicing Raja yoga meditation. This study suggests that the long-term practice of Raja yoga meditation improves basic cardio-respiratory functions due to shifting of the autonomic balance in favour of parasympathetic instead of sympathetic system (Sukhsohale and Phatak, 2012).

\section{References}

Abhishekh H A, Kumar N C, Thirthalli J, Chandrashekar H, Gangadhar B N and Sathyaprabha T N (2014) Prolonged reaction to mental arithmetic stress in first-degree relatives of schizophrenia patients Clin Schizophr Relat Psychoses 8: $137-142$

Adithan C (1996) Pharmacological research in India Indian J Pharmacol 28: $125-128$

Ambarish V, Barde P, Vyas A and Deepak KK (2005) Comparison between pre-prandial and post-prandial heart rate variability (HRV) Indian J Physiol Pharmacol 49: 436442

Bali A and Jaggi A S (2016) An Integrative Review on Role and Mechanisms of Ghrelin in Stress, Anxiety and Depression Curr Drug Targets 17: 495-507

Bansal R, Agarwal M M, Modi M, Mandal A K and Singh S K (2011) Urodynamic profile of diabetic patients with lower urinary tract symptoms: association of diabetic cystopathy with autonomic and peripheral neuropathy Urology 77: 699-705

Bassi A and Bozzali M (2015) Potential Interactions between the Autonomic Nervous System and Higher Level Functions in Neurological and Neuropsychiatric Conditions Front Neurol 6: 1-7

\section{Concluding Remarks}

During the last few decades, implications of abnormal autonomic functions have been explored and its comorbid conditions associated with various cardiovascular, digestive and metabolic disorders are well documented. However, more focus needs to be rendered for the management of comorbid conditions associated with autonomic failures and complications associated with cardiovascular system and neurological disorders along with metabolic disorders. Moreover, emerging research outcome in the field of autonomic nervous system must be a good target for neuropsychiatric and neurodegenerative disorders as well as various cardiovascular, digestive and metabolic disorders. Apart from pharmaco-therapeutic managements of autonomic failures and its complications, modulations of the autonomic nervous system by practice of yoga have been postulated. It is evident from several research that yoga is beneficial in improving the quality of life of an individual suffering from autonomic failures and its complications.

Bhatnagar S, Khanna S, Roshni S, Goyal G N, Mishra S, Rana S $P$ and Thulkar S (2012) Early ultrasound-guided neurolysis for pain management in gastrointestinal and pelvic malignancies: an observational study in a tertiary care center of urban India Pain Pract 12: 23-32

Bhavanani A B, Ramanathan M, Balaji R and Pushpa D (2014) Comparative immediate effect of different yoga asanas on heart rate and blood pressure in healthy young volunteers Int $J$ Yoga 7: 89-95

Chahal S, Vohra K and Syngle A (2017) Association of sudomotor function with peripheral artery disease in type 2 diabetes Neurol Sci 38: 151-156

Chaswal M, Das S, Prasad J, Katyal A and Fahim M (2011) Cardiac autonomic function in acutely nitric oxide deficient hypertensive rats: role of the sympathetic nervous system and oxidative stress Can J Physiol Pharmacol 89: 865874

Chaudhuri A, Borade N G and Hazra S K (2012a) A study of heart rate variablity tests and lipid profile in postmenopausal women J Indian Med Assoc 110: 228, 230-222

Chaudhuri A, Borade N G, Tirumalai J, Saldanha D, Ghosh B and Srivastava K (2012b) A study of autonomic functions and obesity in postmenopausal women Ind Psychiatry $J$ 21: $39-43$ 
Das U N, Repossi G, Dain A and Eynard A R (2011) Is insulin resistance a disorder of the brain? Front Biosci (Landmark Ed) 16: 1-12

Deepika A, Mathew M J, Kumar S A, Devi B I and Shukla D (2015) Paroxysmal sympathetic hyperactivity in pediatric traumatic brain injury: A case series of four patients Auton Neurosci 193: 149-151

Dhargave P, Nalini A, Abhishekh H A, Meghana A, Nagarathna R, Raju T R and Sathyaprabha T N (2014) Assessment of cardiac autonomic function in patients with Duchenne muscular dystrophy using short term heart rate variability measures Eur J Paediatr Neurol 18: 317-320

Fatima N, Pingali U and Pilli R (2014) Evaluation of Phyllanthus emblica extract on cold pressor induced cardiovascular changes in healthy human subjects Pharmacognosy Res 6: 29-35

Goyal R K and Umrani D N (2000) Autonomic Pharmacology Indian J Pharmacol 32: S15-S24

Grassi G and Ram VS (2016) Evidence for a critical role of the sympathetic nervous system in hypertension $J$ Am Soc Hypertens 10: 457-466

Grewal S, Sekhon T S, Walia L and Gambhir R S (2015) Cardiovascular response to acute cold stress in non-obese and obese healthy adults Ethiop J Health Sci 25: 47-52

Hazari M A, Khan R T, Reddy B R and Hassan M A (2012) Cardiovascular autonomic dysfunction in type 2 diabetes mellitus and essential hypertension in a South Indian population Neurosciences (Riyadh) 17: 173-175

Indumathy J, Pal G K, Pal P, Ananthanarayanan P H, Parija S C, Balachander J and Dutta T K (2015) Association of sympathovagal imbalance with obesity indices, and abnormal metabolic biomarkers and cardiovascular parameters Obes Res Clin Pract 9: 55-66

Jangir B L, Mahaprabhu R, Rahangadale S, Bhandarkar A G and Kurkure N V (2016) Neurobehavioral alterations and histopathological changes in brain and spinal cord of rats intoxicated with acrylamide Toxicol Ind Health 32: 526540

Jangpangi D, Mondal S, Bandhu R, Kataria D and Gandhi A (2016) Alteration of Heart Rate Variability in Patients of Depression J Clin Diagn Res 10: Cm04-cm06

Jindal V, Gupta S and Das R (2013) Molecular mechanisms of meditation Mol Neurobiol 48: 808-811

Jyotsna V P, Naseer A, Sreenivas V, Gupta N and Deepak K K (2011) Effect of Cushing's syndrome - Endogenous hypercortisolemia on cardiovascular autonomic functions Auton Neurosci 160: 99-102
Kaushik J S, Chakrabarty B, Gulati S, Patel H, Lodha R, Pai G and Kumar A (2014) Unusual late neurological complication in a child after an Indian krait bite Pediatr Neurol 51: 130132

Krishnamurthy S, Garabadu D and Reddy N R (2013) Asparagus racemosus modulates the hypothalamic-pituitary-adrenal axis and brain monoaminergic systems in rats Nutr Neurosci 16: $255-261$

Krishnamurthy S, Garabadu D, Reddy N R and Joy K P (2011) Risperidone in ultra low dose protects against stress in the rodent cold restraint model by modulating stress pathways Neurochem Res 36: 1750-1758

Krishnaveni G V, Veena S R, Jones A, Srinivasan K, Osmond C, Karat S C, Kurpad A V and Fall C H (2015) Exposure to maternal gestational diabetes is associated with higher cardiovascular responses to stress in adolescent indians $J$ Clin Endocrinol Metab 100: 986-993

Kulshreshtha P and Deepak K K (2013) Autonomic nervous system profile in fibromyalgia patients and its modulation by exercise: a mini review Clin Physiol Funct Imaging 33: 83-91

Kulshreshtha P, Gupta R, Yadav R K, Bijlani R L and Deepak K K (2012a) A comprehensive study of autonomic dysfunction in the fibromyalgia patients Clin Auton Res 22: $117-122$

Kulshreshtha P, Gupta R, Yadav R K, Bijlani R L and Deepak K $\mathrm{K}$ (2012b) Effect of low-dose amitriptyline on autonomic functions and peripheral blood flow in fibromyalgia: a pilot study Pain Med 13: 131-136

Kumar A, Jaryal A, Gulati S, Chakrabarty B, Singh A, Deepak K K, Pandey R M, Gupta N, Sapra S, Kabra M and Khajuria R (2017) Cardiovascular Autonomic Dysfunction in Children and Adolescents With Rett Syndrome Pediatr Neurol 70: 61-66

Kunikullaya K U, Goturu J, Muradi V, Hukkeri PA, Kunnavil R, Doreswamy V, Prakash V S and Murthy N S (2015) Music versus lifestyle on the autonomic nervous system of prehypertensives and hypertensives - a randomized control trial Complement Ther Med 23: 733-740

Meher A, Bhattacharjee M, Rampal P, Kapoor R and Sharma R (2014) Effect of Isometric Exercise on QTc Interval J Clin Diagn Res 8: Bc01-04

Mohan A, Sharma R and Bijlani R L (2011) Effect of meditation on stress-induced changes in cognitive functions $J$ Altern Complement Med 17: 207-212

Muralikrishnan K, Balakrishnan B, Balasubramanian K and Visnegarawla F (2012) Measurement of the effect of Isha Yoga on cardiac autonomic nervous system using short- 
term heart rate variability $J$ Ayurveda Integr Med 3: 91-96

Nagendra H, Kumar V and Mukherjee S (2015) Cognitive behavior evaluation based on physiological parameters among young healthy subjects with yoga as intervention Comput Math Methods Med 2015: 821061

Nayani S, Sreedharan S E, Namboodiri N, Sarma P S and Sylaja P N (2016) Autonomic dysfunction in first ever ischemic stroke: Prevalence, predictors and short term neurovascular outcome Clin Neurol Neurosurg 150: 54-58

Nivethitha L, Mooventhan A and Manjunath N K (2016) Effects of Various Pranayama on Cardiovascular and Autonomic Variables Anc Sci Life 36: 72-77

Pal A, Srivastava N, Narain V S, Agrawal G G and Rani M (2013a) Effect of yogic intervention on the autonomic nervous system in the patients with coronary artery disease: a randomized controlled trial East Mediterr Health J 19: 452-458

Pal G K, Adithan C, Ananthanarayanan P H, Pal P, Nanda N, Durgadevi T, Lalitha V, Syamsunder A N and Dutta T K (2013b) Sympathovagal imbalance contributes to prehypertension status and cardiovascular risks attributed by insulin resistance, inflammation, dyslipidemia and oxidative stress in first degree relatives of type 2 diabetics PLoS One 8: e78072

Pal G K, Adithan C, Ananthanarayanan P H, Pal P, Nanda N, Durgadevi T, Lalitha V, Syamsunder A N and Dutta T K (2014a) Effects of gender on sympathovagal imbalance, prehypertension status, and cardiovascular risks in firstdegree relatives of type 2 diabetics Am J Hypertens 27: 317-324

Pal G K, Chandrasekaran A, Hariharan A P, Dutta T K, Pal P, Nanda N and Venugopal L (2012) Body mass index contributes to sympathovagal imbalance in prehypertensives BMC Cardiovasc Disord 12: 1-9

Pal R, Singh S N, Chatterjee A and Saha M (2014b) Age-related changes in cardiovascular system, autonomic functions, and levels of BDNF of healthy active males: role of yogic practice Age (Dordr) 36: 9683

Pallavi LC, D Souza U J and Shivaprakash G (2017) Assessment of Musculoskeletal Strength and Levels of Fatigue during Different Phases of Menstrual Cycle in Young Adults $J$ Clin Diagn Res 11: Cc11-13

Patel K, Joharapurkar A, Dhanesha N, Patel V, Kshirsagar S, Raval P, Raval S and Jain M R (2014) Thyroid hormone modulates food intake and glycemia via ghrelin secretion in Zucker fatty rats Drug Res (Stuttg) 64: 523-529

Patil P S, Aithala M and Das K K (2015) Effect of Occupational Exposure on Blood Cell Counts, Electrocardiogram and
Blood Pressure in Rice Mill Workers J Clin Diagn Res 9: Cc01-03

Peter R, Sood S and Dhawan A (2015) Spectral Parameters of HRV In Yoga Practitioners, Athletes And Sedentary Males Indian J Physiol Pharmacol 59: 380-387

Rajani S N, Indla Y R, Archana R and Rajesh P (2015) Role of Yoga on Cardic Autonomic Function Tests and Cognition in Type 2 Diabetes Int J Res Ayurveda Pharm 6: 764-766

Rukmani M R, Seshadri S P, Thennarasu K, Raju T R and Sathyaprabha T N (2016) Heart Rate Variability in Children with Attention-Deficit/Hyperactivity Disorder: A Pilot Study Ann Neurosci 23: 81-88

Sahoo D, Kapoor A, Sinha A, Khanna R, Kumar S, Garg N, Tewari S and Goel P (2016) Targeting the sympathoadrenergic link in chronic rheumatic mitral regurgitation: assessing the role of oral beta-blockers Cardiovasc Ther 34: 261-267

Saranya K, Pal G K, Habeebullah S and Pal P (2014) Assessment of cardiovascular autonomic function in patients with polycystic ovary syndrome J Obstet Gynaecol Res 40: 192-199

Sawane M V and Gupta S S (2015) Resting heart rate variability after yogic training and swimming: A prospective randomized comparative trial Int J Yoga 8: 96-102

Senan M and Petrosyan A(2014) Relationship between emotional stress and cardiovascular events Georgian Med News 226: 19-25

Sequeira A and Rosario D (2016) A Comparative Study of Pre and Post-prandial BP Recordings in Obese and Non Obese Young Adults J Clin Diagn Res 10: Oc01-03

Shaikh S I and Verma H (2011) Parkinson's disease and anaesthesia Indian J Anaesth 55: 228-234

Shukla G, Kaul B, Gupta A, Goyal V and Behari M (2015) Parkinsonian syndromes presenting with circadian rhythm sleep disorder- advanced sleep-phase type Natl Med J India 28: 233-235

Sucharita S, Dwarkanath P, Thomas T, Srinivasan K, Kurpad A V and Vaz M (2014) Low maternal vitamin B12 status during pregnancy is associated with reduced heart rate variability indices in young children Matern Child Nutr 10: 226-233

Sukhsohale N D and Phatak M S (2012) Effect of short-term and long-term Brahmakumaris Raja Yoga meditation on physiological variables Indian J Physiol Pharmacol 56: 388-392

Syamsunder A N, Pal P, Pal G K, Kamalanathan C S, Parija S C, Nanda N and Sirisha A (2017) Decreased baroreflex sensitivity is linked to the atherogenic index, retrograde 
inflammation, and oxidative stress in subclinical hypothyroidism Endocr Res 42: 49-58

Syngle A, Verma I, Garg N and Krishan P (2013) Autonomic dysfunction in psoriatic arthritis Clin Rheumatol 32: 10591064

Syngle A, Verma I, Krishan P, Garg N and Syngle V (2015) Disease-modifying anti-rheumatic drugs improve autonomic neuropathy in arthritis: DIANA study Clin Rheumatol 34: 1233-1241

Telles S, Raghavendra B R, Naveen K V, Manjunath N K, Kumar $S$ and Subramanya $P$ (2013) Changes in autonomic variables following two meditative states described in yoga texts $J$ Altern Complement Med 19: 35-42

Telles S and Singh N (2013) Science of the mind: ancient yoga texts and modern studies Psychiatr Clin North Am 36: 93 108

Thakur A K, Chatterjee S S and Kumar V (2015) Adaptogenic potential of andrographolide: An active principle of the king of bitters (Andrographis paniculata) $J$ Tradit Complement Med 5: 42-50

Thakur A K, Soni U K, Rai G, Chatterjee S S and Kumar V (2014) Protective effects of Andrographis paniculata extract and pure andrographolide against chronic stress-triggered pathologies in rats Cell Mol Neurobiol 34: 1111-1121

Thiyagarajan R, Pal P, Pal G K, Subramanian S K, Bobby Z, Das A K and Trakroo M (2013) Cardiovagal modulation, oxidative stress, and cardiovascular risk factors in prehypertensive subjects: cross-sectional study $\mathrm{Am} \mathrm{J}$ Hypertens 26: 850-857

Thiyagarajan R, Subramanian S K, Sampath N, Madanmohan T, Pal P, Bobby Z, Paneerselvam S and Das A K (2012)
Association between cardiac autonomic function, oxidative stress and inflammatory response in impaired fasting glucose subjects: cross-sectional study PLoS One 7 e41889: 41881-41887

Tota S, Kamat P K, Saxena G, Hanif K, Najmi A K and Nath C (2012a) Central angiotensin converting enzyme facilitates memory impairment in intracerebroventricular streptozotocin treated rats Behav Brain Res 226: 317-330

Tota S, Nath C, Najmi A K, Shukla R and Hanif K (2012b) Inhibition of central angiotensin converting enzyme ameliorates scopolamine induced memory impairment in mice: role of cholinergic neurotransmission, cerebral blood flow and brain energy metabolism Behav Brain Res 232: 66-76

Udayabanu M, Kumaran D and Katyal A (2012) Free chelatable zinc modulates the cholinergic function during hypobaric hypoxia-induced neuronal damage: an in vivo study Neuroscience 202: 434-445

Udupa K, Sathyaprabha T N, Thirthalli J, Kishore K R, Lavekar G S, Raju T R and Gangadhar B N (2007) Alteration of cardiac autonomic functions in patients with major depression: a study using heart rate variability measures $J$ Affect Disord 100: 137-141

Vinay A V, Venkatesh D and Ambarish V (2016) Impact of shortterm practice of yoga on heart rate variability Int J Yoga 9: $62-66$

Watve M, Bodas A and Diwekar M (2014) Altered autonomic inputs as a cause of pancreatic beta-cell amyloid Med Hypotheses 82: 49-53

Zope S A and Zope R A (2013) Sudarshan kriya yoga: Breathing for health Int J Yoga 6: 4-10. 